\title{
Activin-A enhances mTOR signaling to promote aberrant chondrogenesis in fibrodysplasia ossificans progressiva
}

\author{
Kyosuke Hino, ${ }^{1,2}$ Kazuhiko Horigome, ${ }^{1,2}$ Megumi Nishio, ${ }^{3}$ Shingo Komura, ${ }^{4,5}$ Sanae Nagata, ${ }^{1}$ Chengzhu Zhao, ${ }^{4}$ Yonghui Jin, ${ }^{3,6}$ \\ Koichi Kawakami,, 8 Yasuhiro Yamada, ${ }^{4,9}$ Akira Ohta, ${ }^{10}$ Junya Toguchida, ${ }^{1,3,6,11}$ and Makoto Ikeya ${ }^{4}$ \\ 'Department of Cell Growth and Differentiation, Center for iPS Cell Research and Application, Kyoto University, Kyoto, Japan. 2iPS Cell-Based Drug Discovery, Sumitomo Dainippon Pharma Co., Ltd., Osaka, \\ Japan. ${ }^{3}$ Department of Tissue Regeneration, Institute for Frontier Life and Medical Sciences, and ${ }^{4}$ Department of Life Science Frontiers, Center for iPS Cell Research and Application, Kyoto University, Kyoto, \\ Japan. ${ }^{5}$ Department of Orthopaedic Surgery, Gifu University Graduate School of Medicine, Gifu, Japan. Institute for Advancement of Clinical and Translational Science (iACT), Kyoto University Hospital, \\ Kyoto, Japan. 'Division of Molecular and Developmental Biology, National Institute of Genetics, Shizuoka, Japan. ${ }^{8}$ Department of Genetics, Graduate University for Advanced Studies (SOKENDAI), Shizuoka, \\ Japan. ${ }^{9}$ Institute for Integrated Cell-Material Sciences (WPI-iCeMS), ${ }^{10}$ Department of Fundamental Cell Technology, Center for iPS Cell Research and Application, and "Department of Orthopaedic Surgery, \\ Graduate School of Medicine, Kyoto University, Kyoto, Japan.
}

Fibrodysplasia ossificans progressiva (FOP) is a rare and intractable disease characterized by extraskeletal bone formation through endochondral ossification. Patients with FOP harbor point mutations in ACVR1, a type I receptor for BMPs. Although mutated ACVR1 (FOP-ACVR1) has been shown to render hyperactivity in BMP signaling, we and others have uncovered a mechanism by which FOP-ACVR1 mistransduces BMP signaling in response to Activin-A, a molecule that normally transduces TCF- $\beta$ signaling. Although Activin-A evokes enhanced chondrogenesis in vitro and heterotopic ossification ( $\mathrm{HO}$ ) in vivo, the underlying mechanisms have yet to be revealed. To this end, we developed a high-throughput screening (HTS) system using FOP patient-derived induced pluripotent stem cells (FOP-iPSCs) to identify pivotal pathways in enhanced chondrogenesis that are initiated by Activin-A. In a screen of 6,809 small-molecule compounds, we identified mTOR signaling as a critical pathway for the aberrant chondrogenesis of mesenchymal stromal cells derived from FOP-iPSCs (FOP-iMSCs). Two different HO mouse models, an FOP model mouse expressing FOP-ACVR1 and an FOP-iPSC-based HO model mouse, revealed critical roles for mTOR signaling in vivo. Moreover, we identified ENPP2, an enzyme that generates lysophosphatidic acid, as a linker of FOP-ACVR1 and mTOR signaling in chondrogenesis. These results uncovered the crucial role of the Activin-A/FOP-ACVR1/ ENPP2/mTOR axis in FOP pathogenesis.

\section{Introduction}

Fibrodysplasia ossificans progressiva (FOP) is a rare genetic disease characterized by extraskeletal bone formation in soft tissue, where bone normally does not exist. Ectopic bones are formed through endochondral ossification, a process in which bone tissue replaces mature cartilage (1-7). Patients with classic FOP have a $617 \mathrm{G}>\mathrm{A}(\mathrm{R} 206 \mathrm{H})$ point mutation in the intracellular glycine- and serine-rich (GS) domain of ACVR1 (8), a type I receptor for BMPs (9-18). This mutation has been shown to render ligandindependent constitutive activity and ligand-dependent hyperactivity in BMP signaling (19-21). The vast majority of drug candidates for FOP treatment suppress the abnormal activation of BMP signaling. Examples include direct kinase inhibitors of the catalytic domain of BMP type I receptors, such as dorsomorphin, LDN-193189, DMH1, RK-0071142, ML357, LDN-212854, and K0288, which consequently suppress the phosphorylation of the downstream effectors SMAD1/5/8 (22-27); RAR $\gamma$ agonists, which

Conflict of interest: K. Hino and K. Horigome are employees of Sumitomo Dainippon Pharma Co., Ltd., and J. Toguchida and M. Ikeya are supported by a research grant from Sumitomo Dainippon Pharma Co., Ltd.

Submitted: February 20, 2017; Accepted: June 13, 2017

Reference information: / Clin Invest. 2017;127(9):3339-3352.

https://doi.org/10.1172/JCI93521. reduce the expression of SMAD1/5/8 by protein degradation (28); allele-specific RNAi (ASP-RNAi) that target mutated ACVR1 (FOPACVR1) mRNA for degradation $(29,30)$; inhibitors of hypoxiainducible factor- $1 \alpha$ (HIF1 $\alpha$ ), which prevent the formation of mesenchymal condensations (31) or amplification of BMP signaling (32); and others $(33,34)$. Among these drug candidates, only palovarotene (28, 35-37), a RAR $\gamma$ agonist, is now in clinical trial.

Recently, we and Hatsell et al. discovered a mechanism of heterotopic ossification (HO) by using FOP patient-derived induced pluripotent stem cells (FOP-iPSCs) and FOP-ACVR1 conditionalon knockin mice $(38,39)$. These studies revealed that mutated FOP-ACVR1 abnormally transduces BMP signaling in response to Activin-A, a molecule that normally transduces TGF- $\beta$ signaling but not BMP signaling. This neofunction of Activin-A raised the possibility that the inhibition of Activin-A signaling by Activin-A-specific-neutralizing antibody or by broad-acting BMP and Activin blockers like ACVR2A-Fc and ACVR2B-Fc (38-42) could be a new treatment strategy for FOP. Although Activin-A evoked enhanced chondrogenesis of induced mesenchymal stromal cells derived from FOP-iPSCs (FOP-iMSCs) via BMP and TGF- $\beta$ signaling $(38,43)$, the detailed mechanism, especially downstream effectors of Activin-A/FOP-ACVR1, is largely unexplained. Therefore, dissecting the molecular mechanisms of this cascade could lead to promising therapeutic strategies. 
Table 1. Source and number of compounds in the HTS library

\begin{tabular}{lcc} 
Library name & Supplier & Number \\
Enzo FDA & Enzo Life Sciences & 636 \\
Enzo ICCB & Enzo Life Sciences & 474 \\
Enzo Kinase Inhibitors & Enzo Life Sciences & 76 \\
LOPAC1280 & Sigma-Aldrich & 1,280 \\
Sigma Pfizer & Sigma-Aldrich & 74 \\
Myriascreen & TimTec & 72 \\
\hline MicroSource International Drugs & MicroSource Discovery Systems & 238 \\
\hline MicroSource US Drugs & MicroSource Discovery Systems & 1,020 \\
EMD Kinase Inhibitors & Merck Millipore & 244 \\
Selleck Kinase Inhibitors & Selleck Chemicals & 141 \\
Tocris Mini Selected for CiRA & Tocris Bioscience & 637 \\
Natural Products & Analyticon Discovery & 1,917 \\
Total & & 6,809
\end{tabular}

To this end, we established an iPSC-based high-throughput screening (HTS) system using FOP-iMSCs and Activin-A-induced enhanced chondrogenesis $(38,44,45)$ and used it to screen our HTS library, which contains approximately 7,000 small-molecule compounds. This screening led us to identify mTOR signaling as a critical downstream pathway of FOP-ACVR1 in enhanced chondrogenesis, an essential step of $\mathrm{HO}$ in the pathology of FOP. Rapamycin, a commercially available drug and commonly used mTOR inhibitor (46-48), showed potent therapeutic effects on HO in 2 different in vivo models triggered by Activin-A: FOP model mice expressing FOP-ACVR1 and an FOP-iPSC-based HO model, in which ectopic bones derived from FOP patient-derived cells are formed in mice. We also identified ENPP2 (also known as autotaxin) as an upstream molecule that positively regulates the mTOR activity triggered by Activin-A. These data uncovered a molecular basis for the $\mathrm{HO}$ induced in patients with FOP.

\section{Results}

Development of an HTS system focused on enhanced chondrogenesis of FOP-iMSCs. Ectopic chondrogenesis is a critical step of endochondral heterotopic ossification in FOP patients (1-6), and enhanced chondrogenesis of FOP-iMSCs was observed when cells were stimulated with Activin-A in vitro and in vivo (38). Therefore, to monitor the degree of enhanced chondrogenesis, an HTS system was established using FOP-iMSCs stably harboring luciferase following 5-repeats Aggrecan enhancers, a well-established chondrogenesis marker $(7,49)$ (FOP-5×A-Luc-iMSCs; Figure 1A). As expected, the induction of chondrogenesis with Activin-A stimulation in a 384-well plate increased luciferase activity in FOP-5×ALuc-iMSCs (Figure 1B). Next, we confirmed the expression level of Aggrecan mRNA (ACAN), the glycosaminoglycan (GAG) production to DNA ratio (GAG/DNA), which represents the extracellular matrix amount of chondrocytes, and Alcian blue staining (acidic polysaccharides such as GAG in chondrocytes) in a 2D chondrogenesis induction (2DCI) assay using FOP-5×A-Luc-iMSCs (Figure $1, \mathrm{C}-\mathrm{E})$. Consistent with the luciferase assay (5×A-Luc assay), Activin-A stimulation increased these values in the 2DCI assay. Therefore, we concluded that the $5 \times A$-Luc assay could be used to

monitor the degree of enhanced chondrogenesis in HTS format (384 wells). Inhibition of either BMP signaling or TGF- $\beta$ signaling with a specific inhibitor (DMH1 or SB-431542, respectively) showed reduced luciferase activity, indicating the pivotal roles of both signaling pathways in chondrogenesis. These data confirmed that the HTS assay we developed can evaluate the enhanced chondrogenesis of FOP-iMSCs stimulated by Activin-A.

HTS and follow-up screens of FOP-iPSCs highlight mTOR signaling as a candidate pathway for modulating enhanced chondrogenesis in FOP. Using this luciferase-based HTS system (5×A-Luc assay), we performed a first screening ( $n=1$; test compounds $=1 \mu \mathrm{M})$ against our HTS library, which contains approximately 7,000 small-molecule compounds, most of which are bioactive and annotated (Figure 2A, Table 1, and Supplemental Figure 1, $\mathrm{A}$ and $\mathrm{B}$; supplemental material available online with this article; https://doi.org/10.1172/JCI93521DS1). The scatter plot distribution (Figure 2B), histogram of raw data (Figure 2C), Z-factor, and signal-to-background (S/B) ratio (Supplemental Figure 1, C and D) confirmed the validity of the HTS campaign. From the first screening, we obtained 549 hit compounds that showed more than $40 \%$ inhibition of luciferase activity against DMSO control cells stimulated with Activin-A. RAR $\gamma$ agonists and dorsomorphin were identified as positive chemicals, indicating the accuracy of our HTS system. A second screening was performed against these 549 compounds ( $n=2$; test compounds $=0.01,0.1$, and $1 \mu \mathrm{M}$ ), and we identified 76 hit compounds that showed an $\mathrm{IC}_{50}$ of less than 1 $\mu \mathrm{M}$ in the $5 \times \mathrm{A}$-Luc assay and low cytotoxicity in the alamarBlue assay (inhibition of $<20 \%$ at any dose) (Supplemental Figures 2 and 3 and Table 2). In the classification of these 76 hit compounds, we found several reported compounds, such as corticosteroids $(22,35)$, RAR agonists $(28,35-37)$, and ALK5 inhibitor (38), that

\section{Table 2. Classification of $\mathbf{7 6}$ hit compounds through the second screening (related to Figure 2)}

$\begin{array}{lc}\text { Category } & \text { Hit number } \\ \text { Corticosteroid } & 15 \\ \text { Natural compound } & 12 \\ \text { DNA topoisomerase inhibitor } & 7 \\ \text { RAR agonist } & 6 \\ \text { mTOR inhibitor } & 5 \\ \text { Typical anticancer } & 5 \\ \text { Reagent } & 5 \\ \text { ALK5 inhibitor } & 4 \\ \text { Src Inhibitor } & 3 \\ \text { Plk inhibitor } & 3 \\ \text { CDK inhibitor } & 2 \\ \text { FCFR inhibitor } & 2 \\ \text { Multi-kinase inhibitor } & 2 \\ \text { ALK inhibitor } & 1 \\ \text { HER2 inhibitor } & 1 \\ \text { PDE inhibitor } & 1 \\ \text { RXR agonist } & 1 \\ \text { Anti-infective agent } & 1 \\ \text { Total } & 76\end{array}$


Table 3. $\mathrm{IC}_{50}$ values for everolimus, rapamycin, and temsirolimus in 5×A-Luc assay, 2DCl assay, and $3 \mathrm{DCl}$ assay (related to Figure 3 )

$\begin{array}{lccc}\mathrm{IC}_{50} & \text { Everolimus } & \text { Rapamycin } & \text { Temsirolimus } \\ 5 \times \mathrm{A} \text {-Luc } & 6.2 \mathrm{nM} & 0.4 \mathrm{nM} & 0.5 \mathrm{nM} \\ 2 \mathrm{DCl} & 1.2 \mathrm{nM} & 0.2 \mathrm{nM} & 0.7 \mathrm{nM} \\ 3 \mathrm{DCl} & 3.1 \mathrm{nM} & 0.5 \mathrm{nM} & 1.1 \mathrm{nM}\end{array}$

were effective for FOP. Among these, we focused on mTOR signaling, because 5 mTOR inhibitors were identified, suggesting the feasibility of mTOR inhibition in this assay system. In particular, rapamycin and its analogs showed potent inhibition in the $5 \times \mathrm{A}$-Luc assay, even at $10 \mathrm{nM}$ (Supplemental Figure 2). Furthermore, the significance of mTOR signaling in FOP pathology has not been well studied.

Next, we performed a detailed concentration-dependent assay against mTOR inhibitors (rapamycin, everolimus, and temsirolimus) using the $5 \times \mathrm{A}-\mathrm{Luc}$ assay. These 3 compounds showed potent $\mathrm{IC}_{50}$ values (Figure 3A and Table 3). To determine whether they actually restore the enhanced chondrogenesis of FOP-iMSCs triggered by Activin-A, we performed 2D and 3D chondrogenesis induction (3DCI) assays. In both assays, these 3 compounds showed potent inhibition of GAG/DNA (Figure 3, B and C, and Table 3), Alcian blue staining (Figure 3, D and E), and expression of chondrogenesis markers (Supplemental Figure 4). Rapamycin showed the strongest $\mathrm{IC}_{50}$ value in these 3 assays; therefore, we performed further analyses mainly using rapamycin. These data indicate that MTOR inhibitors show potent effects on the chondrogenesis of FOP-iMSCs enhanced by Activin-A.

Rapamycin suppresses HO in FOP-ACVR1 conditional transgenic mice. Although rapamycin is reported to prevent both traumainduced and genetic HO (31), its effect on FOP model mice, particularly on mice with Activin-A-induced HO, is unknown. To evaluate the importance of mTOR signaling in FOP model mice, we generated human FOP-ACVR1 (R206H) conditional transgenic mice (Figure 4A and Supplemental Figure 5A). These mice conditionally express FOP-ACVR1 by doxycycline (Dox) administration and are able to escape the perinatal lethality seen in FOP-Acvr1 heterozygous mice (50). Three weeks after Activin-A injection into the right gastrocnemius muscle and induction of FOP-ACVR1 by Dox administration, we observed HO formation in the injected site (Figure 4, B and C, and Table 4). In the injected site, we observed positive staining for safranin $\mathrm{O}$ (acidic proteoglycan, an extracellular matrix protein of chondrocytes), von Kossa (calcium deposition), and COL1 (bone marker), implicating that heterotopic bones were formed through endochondral ossification (Figure 4D). On the contrary, mice administered rapamycin showed little or no heterotopic bone formation. Furthermore, rapamycin administration suppressed the HO triggered by cardiotoxin (Supplemental Figure 5, B-D, and Supplemental Table 1). In these experiments, rapamycin administration did not decrease BW (Supplemental Figure 5, E and F), and the dosing used was comparable to standard rapamycin dosing in humans (51), indicating that the HO suppression might not be mainly caused by toxicity. These rapamycin effects were also confirmed in a BMP-7-induced HO model using WT mice and also with another mTOR inhibitor, everolimus (Supplemental Figure 6 and Supplemental Tables 2 and 3). These results indicated that $\mathrm{mTOR}$ inhibition was effective at suppressing HO in FOP model mice.

Rapamycin suppresses HO derived from FOP-iPSCs in vivo. In both basic and clinical research, species differences are often reported (52-54) and hamper the study of the disease mechanism in humans. To overcome this problem, in vitro and ex vivo assays using patient-derived tissue or iPSCs have been used, but the in vivo assay using human cells remains highly challenging. We previously reported an FOP patient-derived iPSC-based in vivo FOP model, in which ectopic bones derived from FOP-iPSCs were formed in mice (38). In the present study, we evaluated the significance of mTOR signaling in this model. The transplantation of FOP-iMSCs into the gastrocnemius muscle of NOD/SCID mice with C3H1OT1/2 cells harboring Dox-inducible Activin-A showed HO when the mice were administrated Dox (Figure 5, A and B, and Table 5). As with the FOP-ACVR1 conditional transgenic mice, we observed safranin O-, von Kossa-, and COL1-positive cells, suggesting extraskeletal bone formation through an endochondral process (Figure 5C). Interestingly, i.p. or oral administration of rapamycin suppressed the HO triggered by Activin-A (Figure 5, Table 5, Supplemental Figure 7, A and B, and Supplemental Table 4). Since we observed a large number of anti-human-specific vimentin-positive cells in the rapamycin-treated group (Figure 5C), it seemed that the role of rapamycin in this model was not to kill the human cells but rather to suppress $\mathrm{HO}$ itself. Moreover, $\mathrm{HO}$ was suppressed, even when rapamycin treatment was started 2 weeks after transplantation and Dox administration (Supplemental Figure 7, C and D, and Supplemental Table 5); therefore, a window of opportunity for HO suppression would occur not before Activin-A is secreted, but rather after its secretion, when the Activin-A signal has been initiated to some extent. Taken together, rapamycin suppressed FOP-iPSCderived extraskeletal bone formation in vivo, and mTOR inhibition was effective at suppressing HO in both FOP mouse models.

Enhanced mTOR signaling in chondrogenic induction of FOPiMSCs. Although mTOR inhibition was effective at suppressing $\mathrm{HO}$ in vivo, the detailed mechanism was unclear. To clarify the critical role of mTOR signaling and its key components in the chondrogenic induction of FOP-iMSCs, we performed a loss-offunction study using siRNA. MTOR knockdown decreased GAG/ DNA (Figure 6A) and Alcian blue staining (Figure 6B) in 2DCI assays of FOP-iMSCs, demonstrating the essential role of mTOR signaling. It is known that $\mathrm{mTOR}$, a serine/threonine protein kinase, forms the catalytic subunit of 2 different protein com-

\section{Table 4. Number of FOP-ACVR1 conditional transgenic mice harboring $\mathrm{HO}$ ( $>20 \mathrm{~mm}^{3}$ bone volume) triggered by Activin-A (related to Figure 4)}

$\begin{array}{lc}\text { Compound } & \text { No. of } \mathbf{H O s ~}\left(>20 \mathbf{~ m}^{3}\right) \\ \text { Vehicle } & 9 / 11 \\ \text { Rapamycin } & 0 / 11\end{array}$


A

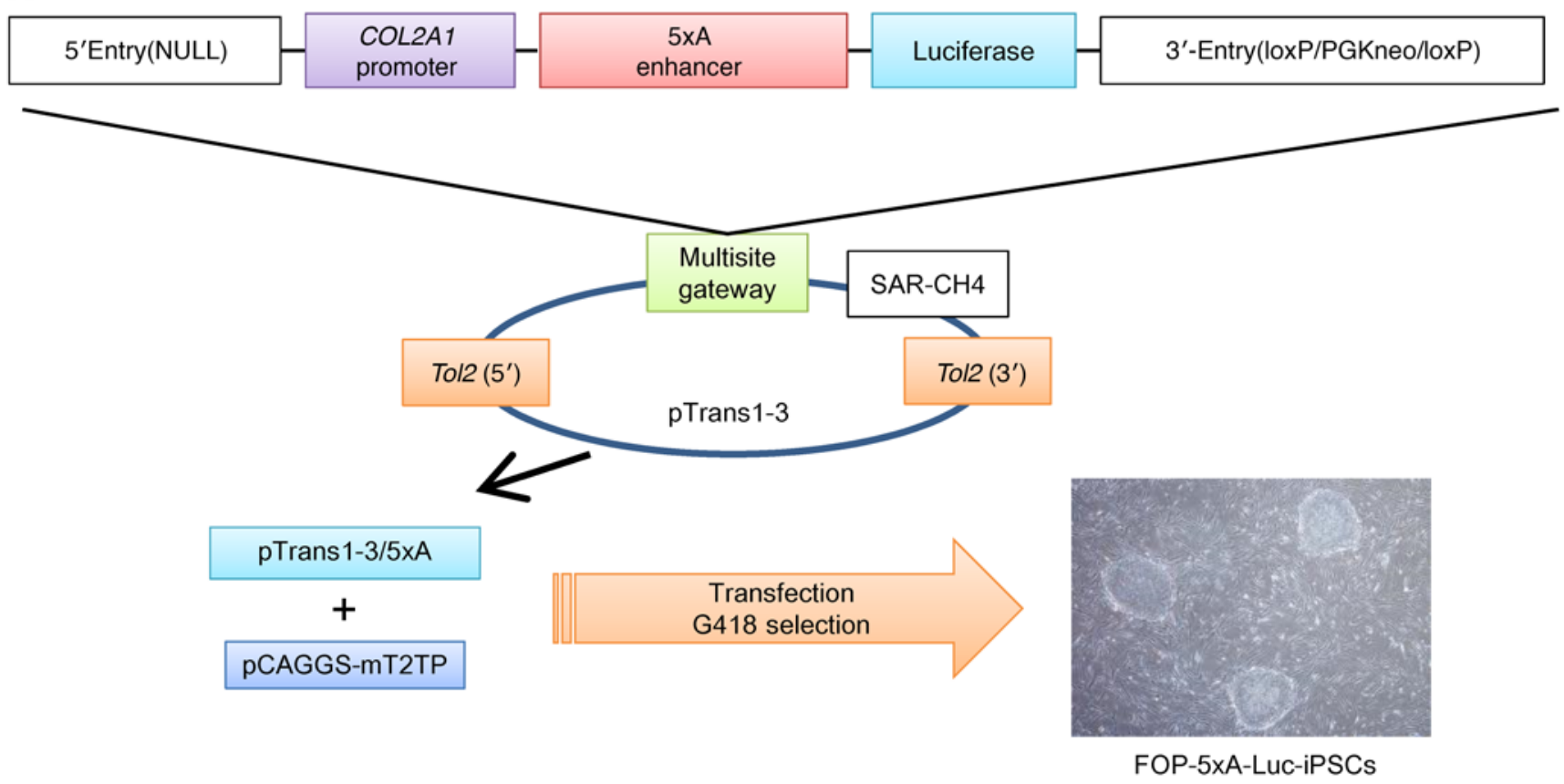

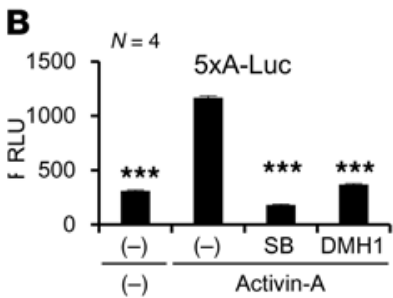

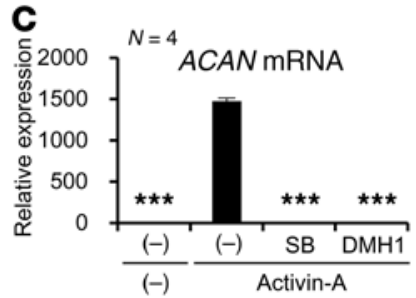

D

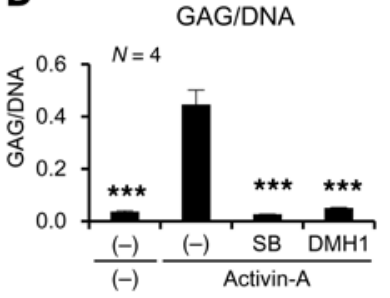

E

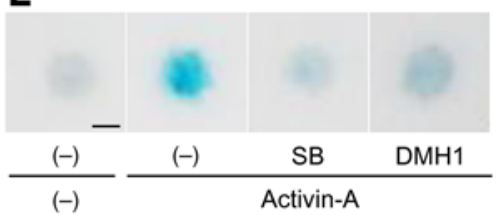

Figure 1. Construction and characterization of the FOP-5×A-Luc assay system. (A) Construction of FOP-5×A-Luc-iPSCs. Luciferase, following the COL2A1 promoter and 5 -repeats Aggrecan enhancer $(5 \times A)$, was inserted into the pTrans1-3 vector to produce stably expressing cell lines by using Tol2 transposase from Japanese medaka fish. After cotransfection with pCAGGS-mT2TP (Tol2 transposase expression vector), the neomycin-resistant clone was selected and designated as FOP-5 $\times$ ALuc-iPSC. (B-E) Characterization of FOP-5×A-Luc-iMSCs. Luciferase activity (B), Aggrecan (ACAN) expression levels (qPCR analysis) (C), GAG/DNA ratios (D), and Alcian blue staining (E) of $2 \mathrm{DCl}$ assays. The cells were harvested 3 days (B), 7 days (C), or 6 days ( $\mathbf{D}$ and $\mathbf{E}$ ) after chondrogenesis induction was performed, with or without Activin-A and inhibitors $(10 \mu \mathrm{M})$. Data represent the mean \pm SEM. $n=4$ (B-D). ${ }^{* *} P<0.001$, by Dunnett's multiple comparisons $t$ test compared with the DMSO treatment control with Activin-A (B-D).Representative data of $n=3$. Scale bar: $200 \mu \mathrm{m}$ (E). SB, SB-43152.

plexes, mTOR complex 1 (mTORC1, a rapamycin-sensitive complex) and mTOR complex 2 (mTORC2, a rapamycin insensitive complex), to orchestrate diverse functions (55-59). Therefore, we analyzed the importance of these complexes in chondrogenesis. As expected, we found that knockdown of RPTOR, a major component of mTORC1, decreased GAG/DNA and Alcian blue staining, but not RICTOR, a major component of mTORC2 (Fig-

Table 5. Number of mice harboring $\mathrm{HO}$ ( $>20 \mathrm{~mm}^{3}$ bone volume) derived from FOP-iPSCs in vivo (related to Figure 5)

\section{Compound}

Vehicle

Rapamycin
No. of $\mathrm{HOs}\left(>20 \mathrm{~mm}^{3}\right)$

$5 / 5$

$0 / 4$

ure 6, A and B). These data indicated a critical role of mTORC1, but not mTORC2, signaling in the 2DCI assays on FOP-iMSCs stimulated with Activin-A. We previously showed that in FOP-iMSCs, Activin-A transduces BMP signaling in addition to TGF- $\beta$ signaling and that inhibition of either signal suppresses enhanced chondrogenesis in FOP-iMSCs (38) (Figure 1, B-E). Therefore, to determine whether rapamycin directly inhibits either BMP or TGF- $\beta$ signaling, we performed Western blotting to analyze the phosphorylation of SMAD1/5/8 (cytoplasmic BMP signaling transducers) and SMAD2/3 (cytoplasmic TGF- $\beta$ signaling transducers). Rapamycin did not directly inhibit BMP (Figure 6C) or TGF- $\beta$ signaling (Figure 6D). Moreover, we found an upregulation of mTORC1 activity triggered by Activin-A during chondrogenesis in FOP-iMSCs compared with that observed in mutation-rescued FOP-iMSCs (resFOP-iMSCs), in which the mutant ACVR1 was converted to WT (45). Phosphorylation of S6 (p-S6), a surrogate marker of mTORC1 activity (55-59), was enhanced in FOP-iMSCs 
A



B
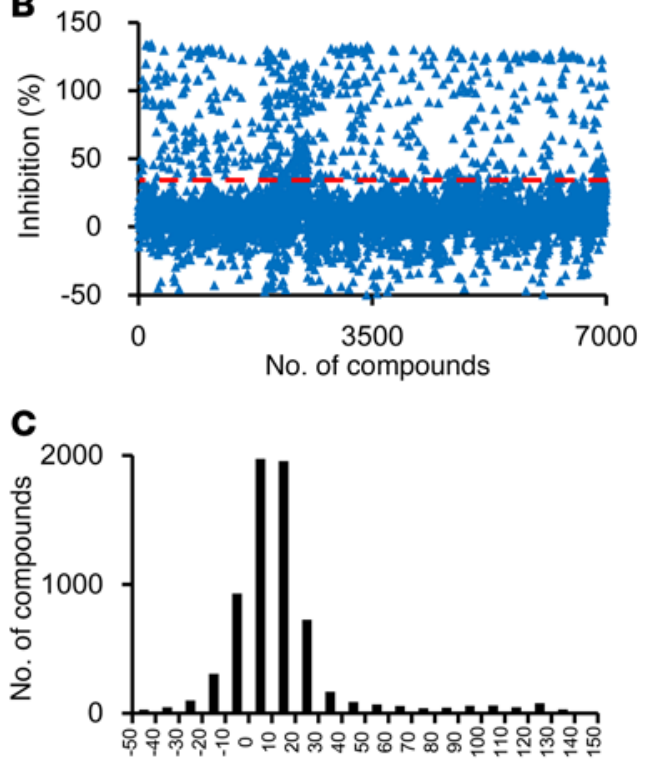

Inhibition (\%)

Figure 2. Identification of mTOR inhibitors through HTS. (A) Schematic of the HTS and follow-up screens. (B and $\mathbf{C}$ ) Raw data from the first screening against 6,809 compounds. Scatter plot distribution (B) and histogram (C) of the percentage of inhibition data. Detailed protocol information and data are provided in Methods and the Supplemental figures.

compared with resFOP-iMSCs stimulated by Activin-A during the 2DCI assay (Figure 6E and Supplemental Figure 8). Finally, to evaluate the signaling cascade of activated mTORC1 signaling, we assessed several inhibitors, including LY294002 (PI3K inhibitor), ipatasertib (AKT inhibitor), and BIRB 796 (p38MAPK inhibitor). In addition to rapamycin, we found that LY294002 and ipatasertib, but not BIRB 796, inhibited the Activin-A-induced phosphorylation of S6 (Figure 6F). These data indicated an involvement of the PI3K/AKT/mTORC1 cascade. Taken together, our findings show that Activin-A activates mTORC1 signaling during chondrogenesis to a greater extent in FOP-iMSCs than in resFOP-iMSCs and that $\mathrm{mTORC} 1$ activity is regulated by PI3K/AKT signaling.

ENPP2-mediated enhanced mTOR signaling in chondrogenic induction of FOP-iMSCs. Next, to gain insight into the molecular mechanisms underlying the enhanced chondrogenesis observed in FOP-iMSCs, we performed an unbiased transcriptome analysis using a DNA microarray. Interestingly, we found that mTOR pathway-related genes were significantly changed in FOP-iMSCs compared with resFOP-iMSCs 24 hours after chondrogenesis induction with Activin-A, but not with BMP-7 or TGF- $\beta 3$ (Supplemental Figure 9). Therefore, Activin-A regulated mTOR signaling in the chondrogenesis of FOP-iMSCs by acting on global gene expression levels. Next, we explored the upstream regulators of mTORC1. Among the genes upregulated in FOP-iMSCs 24 hours after chondrogenesis induction with Activin-A but not with BMP-7 or TGF- $\beta 3$ (Figure 7A), we found that ENPP2 was the most differentially expressed gene (Figure 7B). ENPP2 expression was increased after Activin-A-stimulated chondrogenesis induction, particularly in FOP-iMSCs (Figure 7C). ENPP2 is a secreted lysophospholipase $\mathrm{D}$ that generates the lipid mediator lysophosphatidic acid (LPA), plays a key role in diverse physiological and pathological process-

es, and is known to activate mTOR signaling through LPA receptors $(60,61)$. The administration of ENPP2 inhibitors (HA130 and PF-8380) (Figure 7D) or knockdown of ENPP2 by siRNAs (Figure 7E) decreased the level of p-S6 induced by Activin-A. ENPP2 inhibitors also suppressed GAG/DNA in 2DCI assays of FOP-iMSCs stimulated by Activin-A (Supplemental Figure 10). Conversely, LPA treatment increased p-S6 levels in FOP-iMSCs (Figure $7 F)$. Taken together, these results indicate that ENPP2 is an upstream regulator of the enhanced mTORC1 signaling triggered, at least partially, by Activin-A in FOPiMSCs although further study is required for a more detailed understanding of the underlying mechanism.

\section{Discussion}

In this report, we clarified that mTOR signaling was enhanced in the chondrogenesis of FOP-iMSCs as compared with resFOP-iMSCs and that this aberrant signal was regulated by the Activin-A/FOP-ACVR1/ENPP2 cascade. It is known that mTOR signaling coordinates a variety of environmental inputs including energy, nutrients, stress, and growth factors. Thus, the dysregulation of mTOR signaling, i.e., the abrogation of homeostasis, causes many disorders including cancer, diabetes, neurodegeneration, and others $(47,48,55-59)$. Rodgers et al. proposed that mTORC1 controls tissue regeneration by activating quiescent stem cells into an "alert state" to respond rapidly under conditions of injury and stress $(62,63)$. In FOP pathology, the mutation of ACVR1 appears to deviate this fine-tuned tissue homeostasis mechanism. Activin-A is strongly expressed in wounded regions and promotes the regeneration of damaged tissue (64-66). In FOP, however, we found what we believe to be a new signaling pathway for Activin-A that initiates $\mathrm{HO}$ formation by mistransducing BMP signaling. MSCs, which are cells of origin of $\operatorname{HO}$ in $\operatorname{FOP}(67,68)$, respond abnormally to an Activin-A-evoked signal by taking on an osteo/chondrogenic lineage that is consistent with the response to bone fracture. Because Activin-A activates mTOR signaling in FOP, this response by MSCs could be related to the alert state. Rodgers et al. also showed that HGF signaling is necessary for transition to the alert state as a putative upstream regulator of $\mathrm{MTORC} 1$ signaling. On the other hand, we show that ENPP2-mediated mTORC1 activation is the key event in aberrant chondrogenesis. These observations suggest that mTOR signaling is precisely regulated by the cell type and stimulant. To fully understand mTORC1 signaling in the enhanced chondrogenesis of FOP, it is necessary to investigate the downstream effectors of mTORC1. Furthermore, as we previously discussed (38), because Activin-A does not bind to GREM, an inhibitor of BMPs $(14,16,69)$ that is upregulated in FOP-iMSCs stimulated with Activin-A, BMP signaling does not properly negatively regulate Activin-A. Given these results, we propose that the mechanistic cause of FOP pathology involves the activation of mTOR signaling by Activin-A and the failed negative regulation by BMP signaling.

Although much research has been devoted to mTOR signaling, there are a limited number of reports about the relationship between mTOR signaling and chondrogenesis. During chicken embryonic development, the mechanical activation of mTOR is required for chondrocyte proliferation and chondrogenesis during 
A

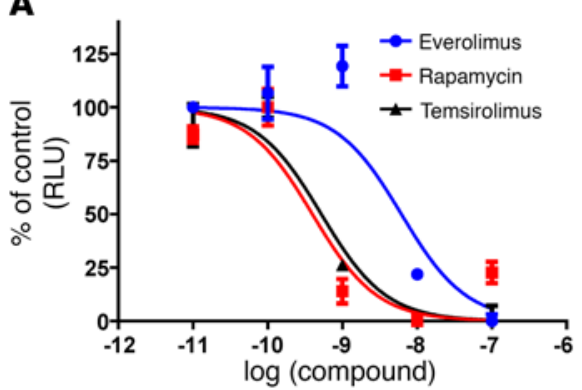

C



B

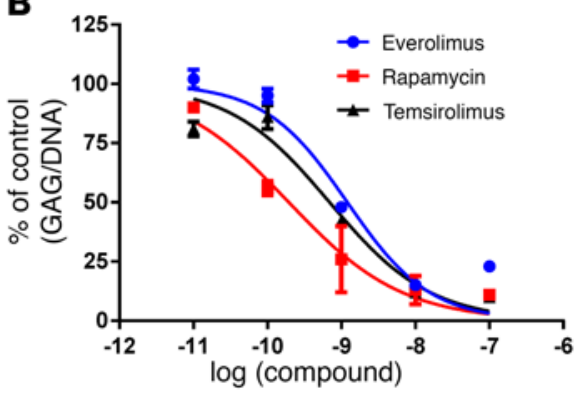

D



Figure 3. mTOR inhibitors suppress the chondrogenic induction of FOP-iMSCs triggered by Activin-A. (AC) Concentration-dependent assays of everolimus, rapamycin, and temsirolimus. $5 \times A$-Luc assay (A), 2DCl assay (B), and 3DCl assay (C) in FOP-iMSCs triggered by Activin-A. Cells were harvested 4 days (A), 7 days (B), or 21 days (C) after chondrogenesis induction was induced with Activin-A, with or without inhibitors. (D and E) Alcian blue staining of FOP-iMSCs in $2 \mathrm{DCl}(\mathbf{D})$ and $3 \mathrm{DCI}(\mathbf{E})$ assays stimulated by Activin-A. Each compound was used at $100 \mathrm{nM}$. Scale bars: $200 \mu \mathrm{m}$ (D) and $100 \mu \mathrm{m}$ (E). Data represent the mean \pm SEM. $n=$ $4(\mathbf{A})$ and $n=3$ (B and $\mathbf{C})$. Results are representative of at least 2 independent experiments.

cyte differentiation (72). These reports suggest that a well-coordinated, but complicated, regulation of mTORC1 activity is crucial for chondrocyte differentiation during development. Further study is needed to clarify the phase of endochondral ossification in which $\mathrm{mTOR}$ signaling is activated and when mTOR signaling should be inhibited to suppress HO. Such investigation will lead to a deeper understanding of FOP pathology and the discovery of new therapeutic targets.

The establishment of an iPSCbased HTS system is one of the features of this study (Figures 1 and 2, Tables 1 and 2, and Supplemental Figures 1-3). Although HTS would be of much greater benefit with patient-derived iPSCs, such an adaptation remains challenging because of unstable protocols and/or long culture periods for differentiation. Although many pathways are involved in chondrogenesis (7), our HTS-based system enabled us to select the pathways crucial for modulating chondrogenesis. We identified mTOR inhibitors as effective drug candidates and confirmed their efficiency with multiple in vivo $\mathrm{HO}$ models (Figures 4 and 5, and Supplemental Figures 5-7). Of note, in one of our HO models, we used human patient-derived FOP cells. This human

bone development (70). In mouse embryos, mTORC1 signaling is activated during limb cartilage development, and the disruption of mTORC1 signaling diminishes embryonic skeletal growth, causing severe delays in chondrocyte hypertrophy and bone formation (71). Furthermore, a reduction in cell size and the amount of cartilage matrix, without changes in chondrocyte proliferation or survival, cause a reduction in the growth of cartilage (71). Both the chicken and mouse studies suggest that mTOR signaling positively modulates chondrogenesis, albeit by distinctive mechanisms. These observations support the notion that mTOR signaling promotes chondrogenesis and are mostly consistent with our study, which showed that mTOR inhibition potently suppressed the enhancement of chondrogenesis in FOP-iMSCs or HO in vivo (Figures 3-5 and Supplemental Figures 4-7). In our 2D and 3DCI assays of Activin-A-stimulated FOP-iMSCs, mTOR inhibitors clearly decreased both GAG/DNA and the expression levels of chondrogenesis markers (Figure 3 and Supplemental Figure 4), indicating that mTOR inhibition did not simply affect proliferation or survival, but modified the differentiation of chondrocytes, since data from both assays were corrected by the number of cells (DNA amount) and the expression of a housekeeping gene ( $\beta$-actin), respectively. On the other hand, another report showed that hyperactivation of mTORC1 via TSC1 gene deletion in chondrocytes blocked chondro-
FOP-iPSC-based in vivo FOP model is unique, in that it enabled us to test the effect of drug candidates on human patient-derived cells in an in vivo environment. However, even though iPSC-based in vivo models have several advantages, there are limitations as well. For example, they require transplantation of the cells into immunodeficient mice, but many types of immune cells contribute to the HO in FOP (73). To ensure the importance of mTOR signaling in FOP pathology, we also established FOP-ACVR1 conditional transgenic mice. This FOP mouse model confirmed the critical role of the mTOR pathway in vivo, although species differences should be considered, since FOP-Acvr1 heterozygous mice showed perinatal lethality (50). Another important aspect of our study is use of a bioactive library that contains currently marketed drugs. The fact that rapamycin is an approved drug (46-48) should accelerate its future clinical trial for FOP. Interestingly, noting the critical roles of Hif1 $\alpha$ on normal chondrogenesis, Agarwal et al. also found that rapamycin decreased extraskeletal bone formation by inhibiting HIF1 $\alpha$ in $3 \mathrm{HO}$ mouse models (31). Also of note, the expression levels of HIF1 $\alpha$ and several related downstream genes were not upregulated in FOP-iMSCs compared with resFOPiMSCs (Supplemental Figure 11), suggesting that the Hif1 a pathway is not related to the Activin-A/FOP-ACVR1/mTOR signaling pathway in FOP-iMSCs, although hypoxia occurs during HO, as dis- 
$\mathbf{A}$

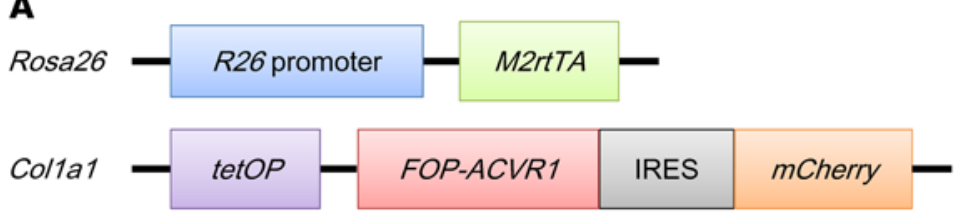

B

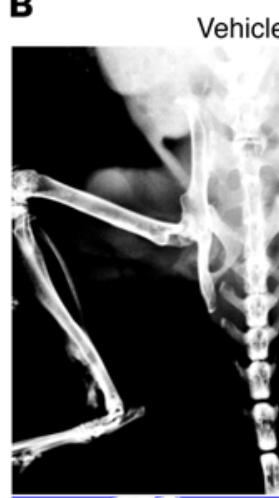

Vehicle
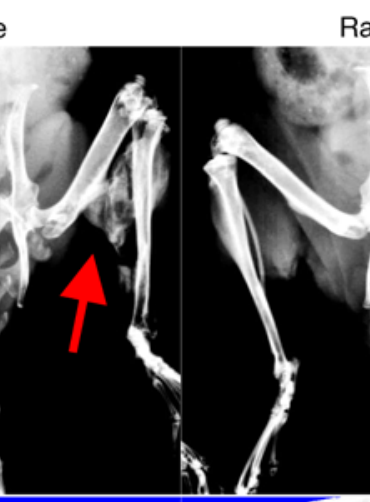

Rapamycin


C



Venicle

D



Figure 4. Rapamycin suppresses Activin-A-triggered HO in FOP-ACVR1 conditional transgenic mice. Schematic of FOP-ACVR1 (R206H) conditional transgenic mice. (B-D) Activin-A injection and oral administration of Dox-induced HO, which was suppressed by i.p. administration of $5 \mathrm{mg} / \mathrm{kg}$ rapamycin (once daily, 5 times a week) in FOP-ACVR1 transgenic mice. The mice were analyzed 3 weeks after Activin-A injection and rapamycin administration. (B) $X$-ray and $\mu \mathrm{CT}$ findings. (C) Average heterotopic bone volume. (D) Histological analysis of the Activin-A-injected region. H\&E, safranin $\mathrm{O}$ (acidic proteoglycan), von Kossa (calcium), and anti-COL1 (bone marker) staining. Scale bars: $10 \mathrm{~mm}$ (B) and $100 \mu \mathrm{m}$ (D). Data represent the mean \pm SEM. $n=11$ (vehicle or rapamycin). ${ }^{* * *} P<0.001$, by Student's $t$ test compared with the vehicle-treated group.

cussed in several studies $(31,32)$. Another important role of mTOR signaling is that of mediating inflammatory responses $(74,75)$, which might have an impact during the early stages of FOP in vivo (73). However, our in vitro chondrogenesis induction experiments using iMSCs without immune cells clearly showed that mTOR signaling is activated downstream of FOP-ACVR1 signaling (Figure 6 and Supplemental Figure 8) and that MTOR inhibitors potently suppressed the enhanced chondrogenesis of FOP-iMSCs (Figure 3 
A



B



c $H \& E$ Safranin O von Kossa

COL1 DAPI
Human vimentin DAPI

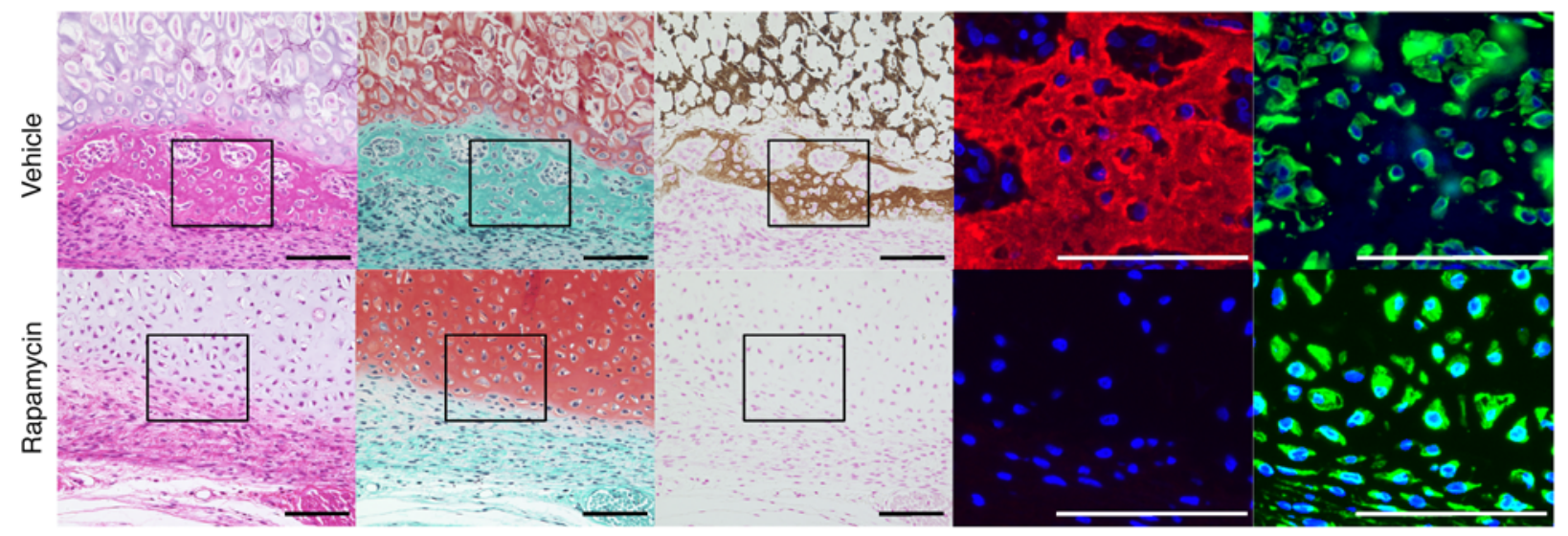

Figure 5. Rapamycin suppresses HO derived from FOP-iPSCs in vivo. Administration of $5 \mathrm{mg} / \mathrm{kg}$ rapamycin (i.p., once daily, 5 times a week) suppressed Activin-A-triggered HO derived from FOP-iMSCs. The mice were analyzed 6 weeks after transplantation and rapamycin administration. (A) X-ray and $\mu \mathrm{CT}$ findings. (B) Average heterotopic bone volume. (C) Histological analysis of the cell-transplanted region. H\&E, safranin O, von Kossa, anti-COL1, and anti-human vimentin staining. Scale bars: $10 \mathrm{~mm}$ (A) and $100 \mu \mathrm{m}$ (C). Results represent the mean \pm SEM. $n=5$ (vehicle) or 4 (rapamycin). ${ }^{* *} P<0.01$, by Student's $t$ test compared with the vehicle-treated group. Data are representative of 3 independent experiments.

and Supplemental Figure 4). We therefore propose that targeting mTOR signaling can modulate aberrant FOP-ACVR1 signaling, hypoxic signaling, and inflammatory signaling during HO. Therefore, mTOR inhibitors have the potential to potently suppress $\mathrm{HO}$ in FOP patients by inhibiting these 3 pathways. Given these serendipitous findings, we believe that rapamycin is a promising drug candidate for the treatment of FOP and that an iPSC-based drug discovery platform, including iPSC-based HTS and in vivo models, offers a remarkable advantage for exploring candidate drugs with a higher probability of reaching clinical trials.

\section{Methods}

Cell culture. iPSCs were maintained in primate embryonic stem (ES) cell medium (ReproCELL) supplemented with $4 \mathrm{ng} / \mathrm{ml}$ recombinant human FGF2 (Wako Pure Chemical). To activate the production of induced neural crest cells (iNCCs), mTeSR1 medium (STEMCELL Technologies) was used for the feeder-free culturing of iPSCs. The induction and maintenance of iNCCs and iMSCs derived from iPSCs were previously described $(43,45)$ (Supplemental Figure 1A). Briefly, iNCCs were induced in chemically defined medium (CDM) supplemented with $10 \mu \mathrm{M}$ SB-431542 and $1 \mu \mathrm{M}$ CHIR99021 for 7 days. iNCCs 

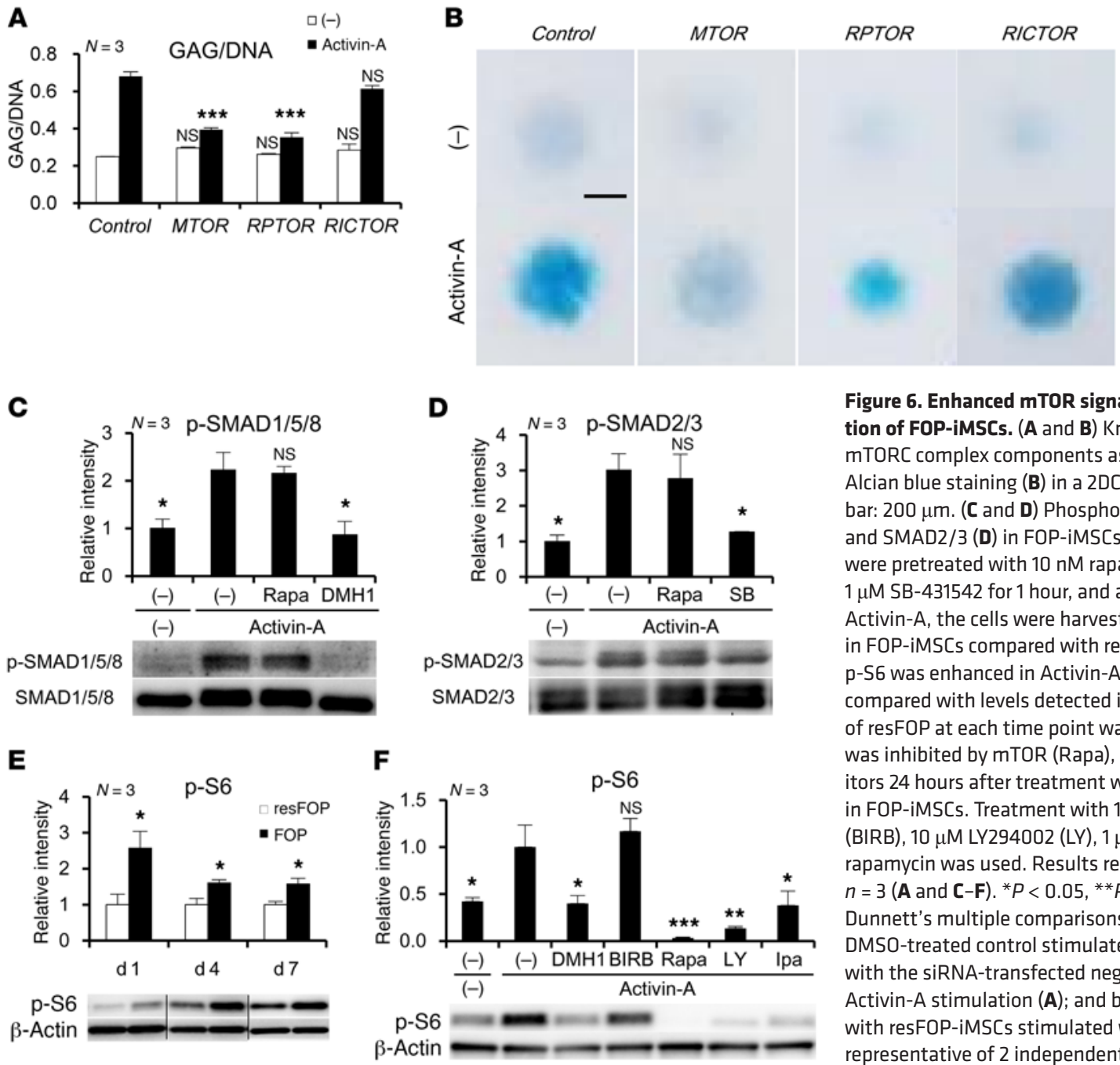

Figure 6. Enhanced mTOR signaling in chondrogenic induction of FOP-iMSCs. (A and B) Knockdown experiments of mTORC complex components assessed by GAG/DNA (A) and Alcian blue staining (B) in a 2DCl assay of FOP-iMSCs. Scale bar: $200 \mu \mathrm{m}$. (C and D) Phosphorylation of SMAD1/5/8 (C) and SMAD2/3 (D) in FOP-iMSCs. Serum-starved FOP-iMSCs were pretreated with $10 \mathrm{nM}$ rapamycin (Rapa), $1 \mu \mathrm{M} \mathrm{DMH1}$, or $1 \mu \mathrm{M}$ SB-431542 for 1 hour, and after a 1-hour stimulation with Activin-A, the cells were harvested. (E) $p-S 6$ was enhanced in FOP-iMSCs compared with resFOP-iMSCs In 2DCl assays, p-S6 was enhanced in Activin-A-stimulated FOP-iMSCs compared with levels detected in resFOP-iMSCs. The strength of resFOP at each time point was set at 1. (F) Enhanced p-S6 was inhibited by mTOR (Rapa), PI3K (LY), or AKT (Ipa) inhibitors 24 hours after treatment with Activin-A and inhibitors in FOP-iMSCs. Treatment with $10 \mu \mathrm{M}$ DMH1, $10 \mu \mathrm{M}$ BIRB 796 (BIRB), $10 \mu \mathrm{M}$ LY294002 (LY), $1 \mu \mathrm{M}$ ipatasertib (Ipa), or $10 \mathrm{nM}$ rapamycin was used. Results represent the mean \pm SEM. $n=3(\mathbf{A}$ and $\mathbf{C}-\mathbf{F}) .{ }^{*} P<0.05,{ }^{* *} P<0.01$, and ${ }^{* *} P<0.001$, by Dunnett's multiple comparisons $t$ test compared with the DMSO-treated control stimulated with Activin-A (C, D, and F); with the siRNA-transfected negative control, with or without Activin-A stimulation (A); and by Student's $t$ test compared with resFOP-iMSCs stimulated with Activin-A (E). Data are representative of 2 independent experiments $(\mathbf{A}, \mathbf{B}, \mathbf{E}$, and $\mathbf{F})$.

were maintained in CDM supplemented with $10 \mu \mathrm{M}$ SB-431542, 20 $\mathrm{ng} / \mathrm{ml} \mathrm{FGF} 2$, and $20 \mathrm{ng} / \mathrm{ml}$ recombinant human EGF (R\&D Systems), and we used up to 20 passages in this study. iMSCs were induced and maintained in $\alpha \mathrm{MEM}$ (Invitrogen, Thermo Fisher Scientific) sup-

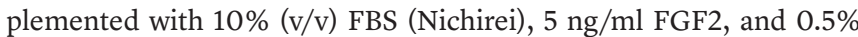
penicillin and streptomycin (Invitrogen, Thermo Fisher Scientific). The FOP-iPSCs used in this study (previously described as vFOP4-1 [ref. 44]) harbor the R206H heterozygous mutation in ACVR1, and gene-corrected resFOP-iPSCs were generated by BAC-based homologous recombination. These cells fulfilled several criteria for iPSCs including the expression of pluripotent markers, teratoma formation, normal karyotype, and morphology. Growth and gene expression profiles of the resFOP-iPSC clones were indistinguishable from the original FOP-iPSCs (45). However, remarkably distinct responsiveness to Activin-A was observed (38). Murine multipotent mesenchymal C3H10T1/2 cells expressing Dox-inducible human INHBA (C3H-DoxOn-hINHBA) were maintained in DMEM (Nacalai Tesque) supplemented with 10\% FBS and $1 \mathrm{mM}$ Na-pyruvate (Thermo Fisher Scientific) and used for an Activin-A-induced HO model transplanted with FOP-iMSCs as previously reported (38).

Reagents. Activin-A, BMP-7, and TGF- $\beta 3$ were purchased from R\&D Systems. Rapamycin and everolimus were purchased from Med-
Chem Express. SB-431542 and temsirolimus were purchased from Sigma-Aldrich. LPA and LY294002 were purchased from Cayman Chemical. Ipatasertib and BIRB 796 were purchased from Selleck Chemicals. DMH1, HA130, and PF-8380 were purchased from Tocris Bioscience, EMD Millipore, and Ark Pharm, respectively. Activin-A, BMP-7, and TGF- $\beta 3$ were dissolved according to the manufacturer's protocols and used at $100 \mathrm{ng} / \mathrm{ml}$ (Activin-A and BMP-7) or $10 \mathrm{ng} / \mathrm{ml}$ (TGF- $\beta 3$ ), unless otherwise noted.

Chemical libraries. All chemical libraries were purchased from the suppliers listed in Table 1. All compounds were bioactive and/or annotated, with the exception of compounds from Analyticon Discovery, which mainly includes natural compounds.

Generation of FOP-5 $\times A-L u c-i P S C s$. Luciferase (luc+; Promega) following the COL2A1 promoter and 5-repeats Aggrecan enhancer (49) was inserted into pTrans1-3 (76), which enabled us to easily produce stably expressing cell lines using Tol2 transposase from Japanese medaka fish (Oryziaslatipes) (77,78) (pTrans1-3/5×A-Luc, Figure1A).pTrans1-3/ $5 \times$ A-Luc and pCAGGS-mT2TP (Tol2 transposase expression vector) were cotransfected into FOP-iPSCs by FuGENE HD (Promega) according to the manufacturer's protocol, and the neomycinresistant clone $(100 \mu \mathrm{g} / \mathrm{ml})$ was selected and used as FOP-5×ALuc-iPSCs. pTrans1-3 included SAR-CH4, an insulator module from 
A

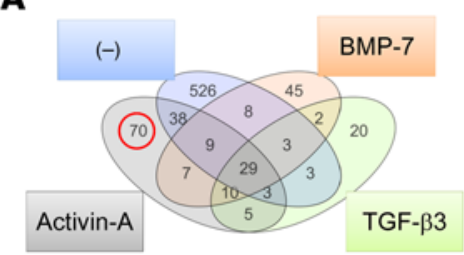

B

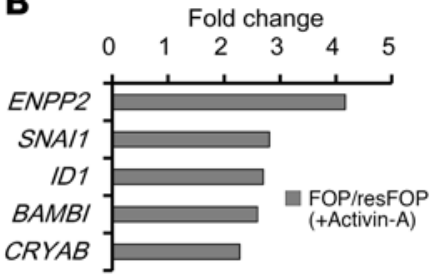

c

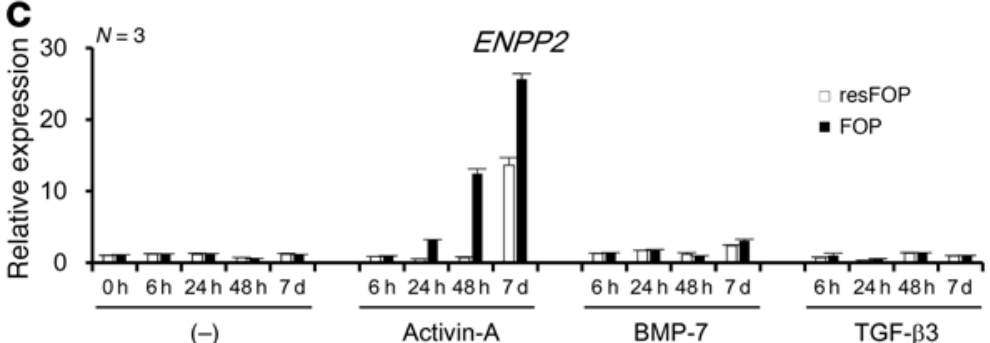

D

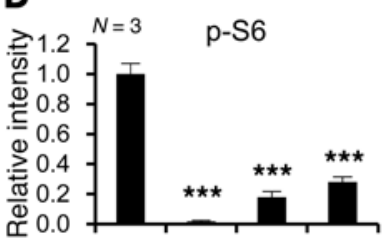

(-) Rapa HA PF



E

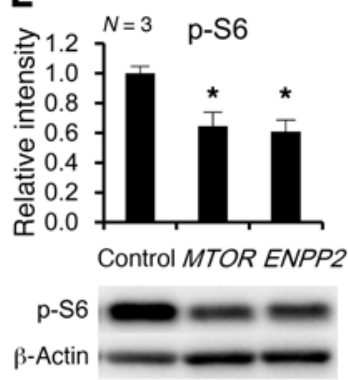

F



(-) LPA

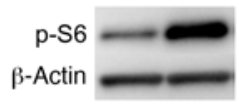

Figure 7. ENPP2 mediates enhanced mTOR signaling in chondrogenic induction of FOPiMSCs. (A) Venn diagram of genes highly upregulated in FOP-iMSCs compared with resFOP-iMSCs 24 hours after chondrogenesis induction stimulated with Activin-A, BMP-7, or TCF- $\beta 3$. (B) Genes highly upregulated in FOP-iMSCs 24 hours after chondrogenesis induction stimulated with Activin-A, but not with BMP-7 or TCF- $\beta 3$. (C) Expression of ENPP2 during the chondrogenic induction of FOP-iMSCs and resFOP-iMSCs. The expression level of resFOP $(0 \mathrm{~h})$ was set at 1. (D and E) ENPP2 inhibitors (HA, HA130; PF, PF-8380) (D) and ENPP2 knockdown (E) reduced p-S6 levels. (D) The cells were harvested 24 hours after treatment with Activin$A$ and ENPP2 inhibitors, and (E) siRNA-transfected cells were harvested 48 hours after treatment with Activin-A. Rapa, 100 nM rapamycin; HA, $10 \mu \mathrm{M} \mathrm{HA130;} \mathrm{PF,} 10 \mu \mathrm{M}$ PF-8380. (F) Serum-starved FOP-iMSCs were treated with $100 \mu \mathrm{M} \mathrm{LPA}$, and 30 minutes after stimulation, the cells were harvested. Results represent the mean \pm SEM. $n=1$ ( $\mathbf{A}$ and $\mathbf{B})$ and $n=3(\mathbf{C}-\mathbf{F})$. ${ }^{*} P<0.05$, ${ }^{*} P<0.01$, and ${ }^{* *} P<0.001$, by Student's $t$ test compared with LPA (-) control $(\mathbf{F})$, by Dunnett's multiple comparisons $t$ test compared with FOP-iMSCs stimulated with ActivinA (D), or with negative control siRNA-transfected FOP-iMSCs stimulated with Activin-A (E). Data are representative of 2 independent experiments ( $\mathbf{D}$ and $\mathbf{F})$.

human IFN- $\beta$ scaffold attachment region and chicken $\beta$-globin DNase I hypersensitive site 4 , and Tol $2\left(5^{\prime}\right)$ or $\left(3^{\prime}\right)$, active transposable Tol 2 elements. pCAGGS-mT2TP is an expression plasmid containing the Tol2 transposase cDNA whose codons are optimized for mammals under the control of the $C A G$ promoter.

HTS. iMSCs differentiated from FOP-5×A-Luc-iPSCs were plated in 384-well white plates (40,000 cells/well/90 $\mu \mathrm{l}$; BD Biosciences) in chondrogenic medium (38) containing $100 \mathrm{ng} / \mathrm{ml}$ Activin-A. After the addition of $10 \mu \mathrm{l}$ of test compounds (final, $1 \mu \mathrm{M}$ ), the assay plates were incubated at $37^{\circ} \mathrm{C}$ under $5 \% \mathrm{CO}_{2}$ (Supplemental Figure 1B). After 4 days of incubation, luciferase activity was measured using the BrightGlo Luciferase Assay System (Promega) according to the manufacturer's protocol. The resultant luminescent signal was measured on PowerScan 4 (DS Pharma Biomedical). The inhibitory effects of the screened compounds are given as the percentage of inhibition, which was calculated using the following equation: (1 - [relative luciferase units (RLU) of compound] - [RLU of minimum [min]]/([RLU of maximum $[\max ]]-[R L U$ of $\min ]) \times 100$, where (RLU of $\max$ ) and (RLU of min) are the mean of the RLU of the DMSO control and $10 \mu \mathrm{M}$ SB-431542, respectively. The Z-factor, which is widely used as a measure of the assay quality of each plate, was calculated using the following equation: Z-factor $=1-3 \times([S D$ of $\max ]+[$ SD of min] $] /([$ RLU of $\max ]-[$ RLU of min $])$ (Supplemental Figure 1C). The average Z-factor over 22 plates in the HTS assay was 0.62, indicating the accuracy and reliability of the HTS campaign (general criteria: HTS >0.50) (79). The S/B ratio was calculated using the following equation: ([RLU of $\max ] /[$ RLU of min]) (Supplemental Figure 1D). The average $\mathrm{S} / \mathrm{B}$ ratio over 22 plates in the HTS assay was 3.9, also indicating the accuracy and reliability of the HTS campaign (general criteria: HTS $>3.0$ ). alamarBlue Cell Viability Reagent (Thermo Fisher Scientific) was used to eliminate toxic compounds in the second screening.

Using the $5 \times \mathrm{A}$-Luc assay, the first screening $(n=1$, $1 \mu \mathrm{M})$ was performed against an HTS library, which contained 6,809 small-molecule compounds. As a result of the first screening, 549 hit compounds were obtained (criteria: $>40 \%$ inhibition of DMSO control stimulated with Activin-A). A second screening was performed against these 549 compounds $(n=2$; test compounds $=0.01,0.1$, and $1 \mu \mathrm{M}$ ), and 76 hit compounds were obtained (criteria: $\mathrm{IC}_{50}<1 \mu \mathrm{M}$ in the $5 \times$ A-Luc assay and inhibition of $<20 \%$ at any dose in the alamarBlue assay). Through the classification of the 76 hit compounds, we focused on mTOR signaling, as mentioned in the Results section.

2DCI and 3DCI. Chondrogenesis induction was performed, and differentiation properties were assayed as previously described $(38,80,81)$. The chondrogenic medium (with $100 \mathrm{ng} / \mathrm{ml}$ Activin-A and inhibitors, unless otherwise noted) was changed every 2 to 3 days until day 7 (2DCI) or until days 14-21 (3DCI), unless otherwise indicated. Genespecific siRNAs were purchased from Thermo Fisher Scientific (Silencer Select Predesigned siRNA). For the transient expression of siRNA, Lipofectamine RNAiMAX Reagent (Thermo Fisher Scientific) was used according to the manufacturer's instructions. Sequence information and knockdown efficiencies are shown in Supplemental Figure 12 and Supplemental Table 6.

Generation of human FOP-ACVR1 conditional transgenic mice. FOP-ACVR1 conditional transgenic mice were generated according to previously reported methods (82-84). Briefly, FOP-ACVR1, followed by IRES-mCherry, was targeted into the 3 '-UTR region of the Col1a1 gene locus under the tetracycline-dependent promoter of $\mathrm{KH} 2$ ES cells, which harbor the optimized reverse tetracycline-dependent transactivator at the ROSA26 locus $(83,84)$. After germline transmission was confirmed, heterozygous ROSA26::M2rtTA mice with a 
heterozygous tetO-FOP-ACVR1 allele were used to induce the FOP$A C V R 1$ gene. Genomic DNA was extracted from the tail of each mouse using a DNeasy Blood and Tissue Kit (QIAGEN) and subjected to genotype analysis. PCR was performed using KOD -Plus- Neo (TOYOBO) according to the manufacturer's instructions. The following PCR primers were used: for Col1a1::FOP-ACVR1: oIMR6724_Col1-FOP, CCTCCATGTGTGACCAAGG; oIMR6725_Col1-FOP， GCACAGCATTGCGGACATGC; and oIMR6726_Col1-FOP, GCAGAAGCGCGGCCGTCTGG; for ROSA26::M2rtTA: oIMR8052_Rosa-tetO, GCGAAGAGTTTGTCCTCAACC; oIMR8545_Rosa-tetO, AAAGTCGCTCTGAGTTGTTAT; and oIMR8546_Rosa-tetO, GGAGCGGGAGAAATGGATATG.

Cardiotoxin-induced HO model in human FOP-ACVR1 conditional transgenic mice. Female mice (filial generations 3-5 [F3-F5] offspring of chimeric mice, age- and BW-matched between groups) were used between 16 and 21 weeks of age. Mice were administered $2 \mathrm{mg} / \mathrm{ml}$ Dox in their drinking water supplemented with $10 \mathrm{mg} / \mathrm{ml}$ sucrose to induce FOP-ACVR1. Cardiotoxin $(9.1 \mu \mathrm{g} /$ mouse; latoxan) was injected into the right gastrocnemius muscle to initiate skeletal muscle injury and subsequent heterotopic bone formation (50). Rapamycin (16\% DMSO in $0.5 \mathrm{w} / \mathrm{v} \%$ methylcellulose $400 \mathrm{cP}$ ) was administered i.p. once a day, 5 times a week. Mice were analyzed 4 weeks after injection. For x-ray images, mice were anesthetized with isoflurane (5\% for induction, 2\%-3\% for maintenance; Abbvie), immobilized, and x-rayed using $\mu \mathrm{FX}-1000$ (Fujifilm) or DX-50 (Faxitron Bioptics). $\mu \mathrm{CT}$ images were scanned using x-ray CT systems (inspeXio SMX-100CT; Shimadzu) and analyzed by TRI/3D-BON software (Ratoc System Engineering) according to the manufacturer's instructions. Four weeks after injection, the injected sites were harvested, fixed with $4 \%$ paraformaldehyde for twenty-four hours, embedded in paraffin, and sectioned and stained with H\&E, von Kossa, safranin O, or anti-collagen I antibody as previously described $(38,80)$.

Activin-A-induced HO model in human FOP-ACVR1 conditional transgenic mice. Male mice (F3-F5 offspring of chimeric mice, age- and BW-matched between groups) were used between 13 and 14 weeks of age. Activin-A ( $13.3 \mu \mathrm{g} /$ mouse) was injected into the right gastrocnemius muscle to initiate skeletal muscle injury and subsequent heterotopic bone formation. Rapamycin (16\% DMSO in $0.5 \mathrm{w} / \mathrm{v} \%$ methylcellulose 400) was administered i.p. once a day, 5 times a week. Mice were analyzed 3 weeks after injection.

BMP-7-induced HO model mice. BMP-7 ( $2 \mu \mathrm{g} /$ mouse $)$ was injected into the right gastrocnemius muscle of 6- to 8-week-old male C57BL/6NJcl mice (CLEA Japan), and compounds were administered once a day, 5 times a week (orally), or every day (i.p.). Mice were analyzed 11-16 days after injection.

Activin-A-induced HO model transplanted with FOP-iMSCs. FOP(right leg) and resFOP-iMSCs (left leg) $\left(4 \times 10^{6}\right.$ cells, respectively) were transplanted into the gastrocnemius muscle of 6- to 8-week-old NOD/ShiJic-scid Jcl (NOD/SCID) mice (CLEA Japan) with C3H-DoxOn-hINHBA $\left(5 \times 10^{5}\right.$ cells $)$, which can continuously expose Activin-A to the transplanted iMSCs in vivo (38). In the Dox-induced group, 1 $\mathrm{mg} / \mathrm{ml}$ Dox (Sigma-Aldrich) was administered via the drinking water with $10 \mathrm{mg} / \mathrm{ml}$ sucrose (Nacalai Tesque) for 2 weeks after transplantation. Compounds were administered i.p. or orally once a day, 5 times a week. Six to eight weeks after transplantation, the transplanted cells were harvested, fixed with $4 \%$ paraformaldehyde for twenty-four hours, embedded in paraffin, and sectioned and stained with H\&E, von Kossa, safranin O, human-specific anti-vimentin antibody, or collagen I antibody as previously described $(38,80,85)$.

Quantitative PCR analysis. Total RNA was purified with an RNeasy Kit (QIAGEN) and treated with a DNase-One Kit (QIAGEN) to remove genomic DNA. Total RNA $(0.3 \mu \mathrm{g})$ was reverse transcribed for singlestranded cDNA using random primers and Superscript III Reverse Transcriptase (Thermo Fisher Scientific) according to the manufacturer's instructions. Quantitative PCR (qPCR) was performed with Thunderbird SYBR qPCR Mix (TOYOBO) and analyzed with the StepOne Real-Time PCR System (Applied Biosystems). The primer sequences are described in Supplemental Table 7. 3D chondrogenic differentiated pellets were homogenized using a Multi-Beads Shocker (Yasui Kikai) according to the manufacturer's instructions. All data (relative expression) were corrected by $\beta$-actin.

Microarray experiments. 2D chondrogenesis induction was performed in FOP- and resFOP-iMSCs stimulated with $100 \mathrm{ng} / \mathrm{ml}$


for 6,24 , or 48 hours or 7 days, mRNA was extracted. RNA was reverse transcribed, biotin labeled, and hybridized to the GeneChip Human Gene 1.0 ST Expression Array (Affymetrix), which was subsequently washed and scanned according to the manufacturer's instructions. Raw CEL files were imported into GeneSpring GX 12.6.1 software (Agilent Technologies), and the expression values were calculated with the RMA16 algorithm. Pathway analysis was performed using Ingenuity Pathway Analysis (QIAGEN). Array data were deposited in the NCBI's Gene Expression Omnibus (GEO) database (GEO GSE90638).

Western blotting. SDS-PAGE and blotting with whole-cell lysates were performed by standard procedures. Protein bands were detected with ECL Prime Western Blotting Detection Reagent (GE Healthcare) and visualized using the Bio-Rad Molecular Imager Chemi-Doc XRS+ with Image Lab software (Bio-Rad). The antibodies used in this study are described in Supplemental Table 8. All data (relative intensity) were corrected by $\beta$-actin or total SMADs.

$G A G$ value. GAG content was quantified in pellets with the Blyscan Glycosaminoglycan Assay Kit (Biocolor). DNA content was quantified using the PicoGreen dsDNA Quantitation Kit (Thermo Fisher Scientific).

IHC. Paraffin-embedded sections were deparaffinized, and for human-specific anti-vimentin antibody, antigen retrieval was performed by autoclaving $\left(105^{\circ} \mathrm{C}, 10 \mathrm{~min}\right)$. Samples were blocked with Blocking One (Nacalai Tesque) for 60 minutes and then incubated with human-specific anti-vimentin antibody (Abcam) or anticollagen I antibody (Novus Biologicals) diluted in Can Get Signal Immunostain Solution B (Toyobo) for 16 to 18 hours at $4^{\circ} \mathrm{C}$. Next, samples were washed several times in 0.2\% Tween-20 (Sigma-Aldrich) in PBS and incubated with goat anti-rabbit $\operatorname{IgG}(\mathrm{H}+\mathrm{L})$ secondary antibody or Alexa Fluor 488 or Alexa Fluor 555 conjugate (Thermo Fisher Scientific) diluted in Can Get Signal Immunostain Solution B for 1 hour at room temperature. DAPI $(10 \mu \mathrm{g} / \mathrm{ml})$ was used to counterstain nuclei. Samples were observed by BZ-9000E (KEYENCE).

Statistics. The statistical significance of all experiments was calculated using 2-tailed Student's $t$ test or 1-way ANOVA for Dunnett's multiple comparisons $t$ test. All statistical tests were performed using GraphPad Prism 6 (GraphPad Software). P values of less than 0.05 were considered statistically significant.

Study approval. All experimental protocols dealing with human subjects were approved by the ethics committee of the Department of Medicine and Graduate School of Medicine of Kyoto University. Written 
informed consent was provided by each donor. All animal experiments were approved by the institutional animal committee of Kyoto University.

\section{Author contributions}

$\mathrm{K}$. Hino, JT, and MI conceived and designed the experiments. $\mathrm{K}$. Hino, K. Horigome, MN, SK, SN, CZ, YJ, and AO performed the experiments. K. Hino, K. Horigome, MN, and AO analyzed the data. K. Horigome, YY, and KK contributed the reagents, materials, and analysis tools. K. Hino, JT, and MI wrote the manuscript. All authors read and approved the final manuscript.

\section{Acknowledgments}

We thank Y. Matsumoto (Hekinan Orthopaedics, Hekinan, Japan) for validating the resFOP iPSCs; M. Shibata (Center for iPS Cell Research and Application, Kyoto University, Kyoto, Japan) for technical assistance; N. Love (RIKEN Center for Developmental Biology, Kobe, Japan) for providing the pTrans1-3 vector; K. Woltjen (Center for iPS Cell Research and Application, Kyoto University, Kyoto, Japan) for providing the PB-TAC-ERN vector; and $\mathrm{H}$. Matsushita (Institute for Frontier Medical Sciences, Kyoto University, Kyoto, Japan) and staff at the Center for Anatomical, Pathological and Forensic Medical Research of the Kyoto University Graduate School of Medicine for preparing the microscope slides. We thank A. Yamashita (Center for iPS Cell Research and Application, Kyoto University, Kyoto, Japan) for advice on immunostaining; R. Nakayama (Sumitomo Dainippon Pharma Co., Ltd., Osaka, Japan) for advice on in vivo experiments; K. Eto, C. Alev, (Center for iPS Cell Research and Application, Kyoto University, Kyoto, Japan) and A. Ikeda (Sumitomo Dainippon Pharma Co., Ltd., Osaka, Japan) for invaluable comments and discussion; P. Karagiannis (Center for iPS Cell Research and Application, Kyoto University, Kyoto, Japan) for reading the manuscript; S. Yamanaka (Center for iPS Cell Research and Application, Kyoto Universi- ty, Kyoto, Japan) for supporting and initiating our FOP research; and members of the JT and MI laboratories for their support throughout this study. This work was supported by Grants-in-Aid for Scientific Research from the Japan Society for the Promotion of Science (JSPS) (25293320 and 16K15662); the Leading Project for Realization of Regenerative Medicine from the Japanese Ministry of Education, Culture, Sports, Science and Technology (MEXT); the Program for Intractable Diseases Research Utilizing Disease-Specific iPS Cells, from the Japan Science and Technology Agency (JST) and the Japan Agency for Medical Research and Development (AMED); the Core Center for iPS Cell Research of the Research Center Network for the Realization of Regenerative Medicine (JST/AMED); a grant from Research on Development of New Drugs (AMED); and a grant from the iPS Cell Research Fund, awarded in part to MI and JT. MI was also supported by a grant from A-STEP (Adaptable and Seamless Technology Transfer Program Through Target-Driven R\&D, Exploratory Research) from the JST (AS242Z00931P) and the Practical Research Project for Rare/Intractable Diseases from AMED. CZ was also supported by Grant-in-Aid for Young Scientists B from JSPS (17K15617). AO was also supported by Research Project for Practical Applications of Regenerative Medicine from AMED. These funders had no role in the study design, data collection and analysis, decision to publish, or preparation of the manuscript.

Address correspondence to: Makoto Ikeya, Department of Life Science Frontiers, Center for iPS Cell Research and Application, Kyoto University, 53 Kawahara-cho, Shogoin, Sakyo-ku, Kyoto, 6068507, Japan. Phone: 81.75.366.7054; Email: mikeya@cira.kyoto-u. ac.jp. Or to: Junya Toguchida, Department of Tissue Regeneration, Institute for Frontier Life and Medical Sciences, Kyoto University, 53 Kawahara-cho, Shogoin, Sakyo-ku, Kyoto, 606-8507, Japan. Phone: 81.75.751.4134; Email: togjun@frontier.kyoto-u.ac.jp.
1. Kaplan F, et al. The phenotype of fibrodysplasia ossificans progressiva. Clinic Rev Bone Miner Metab. 2005;3:(3-4):183-188.

2. Shore E, Feldman G, Xu M, Kaplan F. The genetics of fibrodysplasia ossificans progressiva. Clinic Rev Bone Miner Metab. 2005;3(346):201-204.

3. Kaplan FS, Groppe J, Pignolo RJ, Shore EM. Morphogen receptor genes and metamorphogenes: skeleton keys to metamorphosis. Ann N Y Acad Sci. 2007;1116:113-133.

4. Kaplan FS, et al. Fibrodysplasia ossificans progressiva. Best Pract Res Clin Rheumatol. 2008;22(1):191-205.

5. Shore EM, Kaplan FS. Inherited human diseases of heterotopic bone formation. Nat Rev Rheumatol. 2010;6(9):518-527.

6. Kaplan FS, Chakkalakal SA, Shore EM. Fibrodysplasia ossificans progressiva: mechanisms and models of skeletal metamorphosis. Dis Model Mech. 2012;5(6):756-762.

7. Zuscik MJ, Hilton MJ, Zhang X, Chen D, O'Keefe RJ. Regulation of chondrogenesis and chondrocyte differentiation by stress. J Clin Invest. 2008;118(2):429-438.

8. Shore EM, et al. A recurrent mutation in the BMP type I receptor ACVR1 causes inherited and sporadic fibrodysplasia ossificans progressiva. $\mathrm{Nat}$ Genet. 2006;38(5):525-527.

9. Urist MR. Bone: formation by autoinduction. Science. 1965;150(3698):893-899.

10. Hogan BL. Bone morphogenetic proteins: multifunctional regulators of vertebrate development. Genes Dev. 1996;10(13):1580-1594.

11. Wozney JM, et al. Novel regulators of bone formation: molecular clones and activities. Science. 1988;242(4885):1528-1534.

12. Gu Z, et al. The type I serine/threonine kinase receptor ActRIA (ALK2) is required for gastrulation of the mouse embryo. Development. 1999;126(11):2551-2561.

13. Mishina Y, Crombie R, Bradley A, Behringer RR. Multiple roles for activin-like kinase-2 signaling during mouse embryogenesis. Dev Biol. 1999;213(2):314-326.

14. Piek E, Heldin CH, Ten Dijke P. Specificity, diversity, and regulation in TGF-beta superfamily signaling. FASEB J. 1999;13(15):2105-2124.

15. Massagué J, Blain SW, Lo RS. TGFbeta signaling in growth control, cancer, and heritable disorders. Cell. 2000;103(2):295-309.
16. Canalis E, Economides AN, Gazzerro E. Bone morphogenetic proteins, their antagonists, and the skeleton. Endocr Rev. 2003;24(2):218-235.

17. Miyazono K, Kamiya Y, Morikawa M. Bone morphogenetic protein receptors and signal transduction. J Biochem. 2010;147(1):35-51.

18. Mueller TD, Nickel J. Promiscuity and specificity in BMP receptor activation. FEBS Lett. 2012;586(14):1846-1859.

19. Fukuda T, et al. A unique mutation of ALK2, G356D, found in a patient with fibrodysplasia ossificans progressiva is a moderately activated BMP type I receptor. Biochem Biophys Res Commun. 2008;377(3):905-909.

20. Billings PC, et al. Dysregulated BMP signaling and enhanced osteogenic differentiation of connective tissue progenitor cells from patients with fibrodysplasia ossificans progressiva (FOP). JBone Miner Res. 2008;23(3):305-313.

21. Chaikuad A, et al. Structure of the bone morphogenetic protein receptor ALK2 and implications for fibrodysplasia ossificans progressiva. J Biol Chem. 2012;287(44):36990-36998.

22. Yu PB, et al. BMP type I receptor inhibition reduces heterotopic [corrected] ossification. Nat Med. 
2008;14(12):1363-1369.

23. Hao J, et al. In vivo structure-activity relationship study of dorsomorphin analogues identifies selective VEGF and BMP inhibitors. ACS Chem Biol. 2010;5(2):245-253.

24. Hamasaki M, et al. Pathogenic mutation of ALK2 inhibits induced pluripotent stem cell reprogramming and maintenance: mechanisms of reprogramming and strategy for drug identification. Stem Cells. 2012;30(11):2437-2449.

25. Engers DW, Frist AY, Lindsley CW, Hong CC, Hopkins CR. Synthesis and structure-activity relationships of a novel and selective bone morphogenetic protein receptor (BMP) inhibitor derived from the pyrazolo[1.5-a]pyrimidine scaffold of dorsomorphin: the discovery of ML347 as an ALK2 versus ALK3 selective MLPCN probe. Bioorg Med Chem Lett. 2013;23(11):3248-3252.

26. Mohedas AH, Xing X, Armstrong KA, Bullock AN, Cuny GD, Yu PB. Development of an ALK2-biased BMP type I receptor kinase inhibitor. ACS Chem Biol. 2013;8(6):1291-1302.

27. Sanvitale CE, et al. A new class of small molecule inhibitor of BMP signaling. PLoS One. 2013;8(4):e62721.

28. Shimono K, et al. Potent inhibition of heterotopic ossification by nuclear retinoic acid receptor- $\gamma$ agonists. Nat Med. 2011;17(4):454-460.

29. Kaplan J, Kaplan FS, Shore EM. Restoration of normal BMP signaling levels and osteogenic differentiation in FOP mesenchymal progenitor cells by mutant allele-specific targeting. Gene Ther. 2012;19(7):786-790.

30. Takahashi M, Katagiri T, Furuya H, Hohjoh H. Disease-causing allele-specific silencing against the ALK2 mutants, R206H and G356D, in fibrodysplasia ossificans progressiva. Gene Ther. 2012;19(7):781-785.

31. Agarwal S, et al. Inhibition of Hifl $\alpha$ prevents both trauma-induced and genetic heterotopic ossification. Proc Natl Acad Sci USA. 2016;113(3):E338-E347.

32. Wang H, et al. Cellular Hypoxia Promotes Heterotopic Ossification by Amplifying BMP Signaling. J Bone Miner Res. 2016;31(9):1652-1665.

33. Kitoh $\mathrm{H}$, et al. Perhexiline maleate in the treatment of fibrodysplasia ossificans progressiva: an open-labeled clinical trial. Orphanet J Rare Dis. 2013;8:163.

34. Cappato S, et al. High-throughput screening for modulators of ACVR1 transcription: discovery of potential therapeutics for fibrodysplasia ossificans progressiva. Dis Model Mech. 2016;9(6):685-696.

35. Sinha S, Uchibe K, Usami Y, Pacifici M, Iwamoto M. Effectiveness and mode of action of a combination therapy for heterotopic ossification with a retinoid agonist and an anti-inflammatory agent. Bone. 2016;90:59-68.

36. Pavey GJ, et al. Targeted stimulation of retinoic acid receptor- $\gamma$ mitigates the formation of heterotopic ossification in an established blast-related traumatic injury model. Bone. 2016;90:159-167.

37. Chakkalakal SA, et al. Palovarotene Inhibits Heterotopic Ossification and Maintains Limb Mobility and Growth in Mice With the Human ACVR1(R206H) Fibrodysplasia Ossificans Progressiva (FOP) Mutation. JBone Miner Res.
2016;31(9):1666-1675.

38. Hino K, et al. Neofunction of ACVR1 in fibrodysplasia ossificans progressiva. Proc Natl Acad Sci USA. 2015;112(50):15438-15443.

39. Hatsell SJ, et al. ACVR1R206H receptor mutation causes fibrodysplasia ossificans progressiva by imparting responsiveness to activin A. Sci Transl Med. 2015;7(303):303ra137.

40. del Re E, Sidis Y, Fabrizio DA, Lin HY, Schneyer A. Reconstitution and analysis of soluble inhibin and activin receptor complexes in a cell-free system. J Biol Chem. 2004;279(51):53126-53135.

41. Souza TA, et al. Proteomic identification and functional validation of activins and bone morphogenetic protein 11 as candidate novel muscle mass regulators. Mol Endocrinol. 2008;22(12):2689-2702.

42. Sako D, et al. Characterization of the ligand binding functionality of the extracellular domain of activin receptor type IIb. J Biol Chem. 2010;285(27):21037-21048.

43. Fukuta M, et al. Derivation of mesenchymal stromal cells from pluripotent stem cells through a neural crest lineage using small molecule compounds with defined media. PLoS One. 2014;9(12):e112291.

44. Matsumoto Y, et al. Induced pluripotent stem cells from patients with human fibrodysplasia ossificans progressiva show increased mineralization and cartilage formation. Orphanet J Rare Dis. 2013;8:190.

45. Matsumoto Y, et al. New Protocol to Optimize iPS Cells for Genome Analysis of Fibrodysplasia Ossificans Progressiva. Stem Cells. 2015;33(6):1730-1742.

46. Vézina C, Kudelski A, Sehgal SN. Rapamycin (AY$22,989)$, a new antifungal antibiotic. I. Taxonomy of the producing streptomycete and isolation of the active principle. J Antibiot. 1975;28(10):721-726.

47. Benjamin D, Colombi M, Moroni C, Hall MN Rapamycin passes the torch: a new generation of mTOR inhibitors. Nat Rev Drug Discov. 2011;10(11):868-880.

48. Vakifahmetoglu-Norberg H, Xia HG, Yuan J. Pharmacologic agents targeting autophagy. JClin Invest. 2015;125(1):5-13.

49. Han Y, Lefebvre V. L-Sox5 and Sox6 drive expression of the aggrecan gene in cartilage by securing binding of Sox 9 to a far-upstream enhancer. $\mathrm{Mol}$ Cell Biol. 2008;28(16):4999-5013.

50. Chakkalakal SA, et al. An Acvr1 R206H knock-in mouse has fibrodysplasia ossificans progressiva. J Bone Miner Res. 2012;27(8):1746-1756.

51. Lee N, Woodrum CL, Nobil AM, Rauktys AE, Messina MP, Dabora SL. Rapamycin weekly maintenance dosing and the potential efficacy of combination sorafenib plus rapamycin but not atorvastatin or doxycycline in tuberous sclerosis preclinical models. BMC Pharmacol. 2009;9:8.

52. Ebert AD, Svendsen CN. Human stem cells and drug screening: opportunities and challenges. Nat Rev Drug Discov. 2010;9(5):367-372.

53. Grskovic M, Javaherian A, Strulovici B, Daley GQ. Induced pluripotent stem cells--opportunities for disease modelling and drug discovery. Nat Rev Drug Discov. 2011;10(12):915-929.

54. Merkle FT, Eggan K. Modeling human disease with pluripotent stem cells: from genome association to function. Cell Stem Cell. 2013;12(6):656-668.

55. Ma XM, Blenis J. Molecular mechanisms of mTOR-mediated translational control. Nat Rev Mol Cell Biol. 2009;10(5):307-318.

56. Laplante M, Sabatini DM. mTOR signaling in growth control and disease. Cell. 2012;149(2):274-293.

57. Johnson SC, Rabinovitch PS, Kaeberlein M. mTOR is a key modulator of ageing and agerelated disease. Nature. 2013;493(7432):338-345.

58. Shimobayashi M, Hall MN. Making new contacts: the mTOR network in metabolism and signalling crosstalk. Nat Rev Mol Cell Biol. 2014;15(3):155-162.

59. Saxton RA, Sabatini DM. mTOR Signaling in Growth, Metabolism, and Disease. Cell. 2017;168(6):960-976.

60. Winter JN, Fox TE, Kester M, Jefferson LS, Kimball SR. Phosphatidic acid mediates activation of mTORC1 through the ERK signaling pathway. Am JPhysiol, Cell Physiol. 2010;299(2):C335-C344.

61. Samadi N, Bekele R, Capatos D, Venkatraman G, Sariahmetoglu M, Brindley DN. Regulation of lysophosphatidate signaling by autotaxin and lipid phosphate phosphatases with respect to tumor progression, angiogenesis, metastasis and chemo-resistance. Biochimie. 2011;93(1):61-70.

62. Rodgers JT, et al. mTORC1 controls the adaptive transition of quiescent stem cells from G0 to G(Alert). Nature. 2014;510(7505):393-396.

63. Cheung TH, Rando TA. Molecular regulation of stem cell quiescence. Nat Rev Mol Cell Biol. 2013;14(6):329-340.

64. Cruise BA, Xu P, Hall AK. Wounds increase activin in skin and a vasoactive neuropeptide in sensory ganglia. Dev Biol. 2004;271(1):1-10.

65. Antsiferova M, Werner S. The bright and the dark sides of activin in wound healing and cancer. J Cell Sci. 2012;125(Pt 17):3929-3937.

66. Aleman-Muench GR, Soldevila G. When versatility matters: activins/inhibins as key regulators of immunity. Immunol Cell Biol. 2012;90(2):137-148.

67. Agarwal S, et al. Scleraxis-Lineage Cells Contribute to Ectopic Bone Formation in Muscle and Tendon. Stem Cells. 2017;35(3):705-710.

68. Dey D, et al. Two tissue-resident progenitor lineages drive distinct phenotypes of heterotopic ossification. Sci Transl Med. 2016;8(366):366ra163.

69. Hsu DR, Economides AN, Wang X, Eimon PM, Harland RM. The Xenopus dorsalizing factor Gremlin identifies a novel family of secreted proteins that antagonize BMP activities. Mol Cell. 1998;1(5):673-683.

70. Guan Y, Yang X, Yang W, Charbonneau C, Chen Q. Mechanical activation of mammalian target of rapamycin pathway is required for cartilage development. FASEB J. 2014;28(10):4470-4481.

71. Chen J, Long F. mTORC1 signaling controls mammalian skeletal growth through stimulation of protein synthesis. Development. 2014;141(14):2848-2854.

72. Yan B, et al. mTORC1 regulates PTHrP to coordinate chondrocyte growth, proliferation and differentiation. Nat Commun. 2016;7:11151.

73. Kaplan FS, Pignolo RJ, Shore EM. Granting immunity to FOP and catching heterotopic ossification in the Act. Semin Cell Dev Biol. 2016;49:30-36 
74. Thomson AW, Turnquist HR, Raimondi G. Immunoregulatory functions of mTOR inhibition. Nat Rev Immunol. 2009;9(5):324-337.

75. Weichhart T, Hengstschläger M, Linke M. Regulation of innate immune cell function by mTOR. Nat Rev Immunol. 2015;15(10):599-614.

76. Love NR, et al. pTransgenesis: a cross-species, modular transgenesis resource. Development. 2011;138(24):5451-5458.

77. Tsukahara T, et al. The Tol2 transposon system mediates the genetic engineering of T-cells with CD19-specific chimeric antigen receptors for B-cell malignancies. Gene Ther. 2015;22(2):209-215.

78. Kawakami K, Noda T. Transposition of the Tol2 element, an Ac-like element from the Japanese medaka fish Oryzias latipes, in mouse embryonic stem cells. Genetics. 2004;166(2):895-899.

79. Zhang JH, Chung TD, Oldenburg KR. A Simple Statistical Parameter for Use in Evaluation and Validation of High Throughput Screening Assays. J Biomol Screen. 1999;4(2):67-73.

80. Umeda K, Zhao J, Simmons P, Stanley E, Elefanty A, Nakayama N. Human chondrogenic paraxial mesoderm, directed specification and prospective isolation from pluripotent stem cells. Sci Rep. 2012;2:455.

81. Nasu A, et al. Genetically matched human iPS cells reveal that propensity for cartilage and bone differentiation differs with clones, not cell type of origin. PLoS One. 2013;8(1):e53771.

82. Beard C, Hochedlinger K, Plath K, Wutz A, Jaenisch R. Efficient method to generate single-copy transgenic mice by site-specific integration in embryonic stem cells. Genesis. 2006;44(1):23-28.

83. Yamada K, et al. EWS/ATF1 expression induces sarcomas from neural crest-derived cells in mice. JClin Invest. 2013;123(2):600-610.

84. Ohnishi K, et al. Premature termination of reprogramming in vivo leads to cancer development through altered epigenetic regulation. Cell. 2014;156(4):663-677.

85. Yamashita A, et al. Generation of scaffoldless hyaline cartilaginous tissue from human iPSCs. Stem Cell Reports. 2015;4(3):404-418. 